\title{
Bisphenyl-Polymer/Carbon-Fiber-Reinforced Composite Compared to Titanium Alloy Bone Implant
}

\author{
Richard C. Petersen \\ Department of Biomaterials, University of Alabama at Birmingham, Birmingham, AL 35294-0007, USA \\ Correspondence should be addressed to Richard C. Petersen, richbme@uab.edu
}

Received 21 March 2011; Revised 22 April 2011; Accepted 3 May 2011

Academic Editor: Shanfeng Wang

Copyright () 2011 Richard C. Petersen. This is an open access article distributed under the Creative Commons Attribution License, which permits unrestricted use, distribution, and reproduction in any medium, provided the original work is properly cited.

\begin{abstract}
Aerospace/aeronautical thermoset bisphenyl-polymer/carbon-fiber-reinforced composites are considered as new advanced materials to replace metal bone implants. In addition to well-recognized nonpolar chemistry with related bisphenol-polymer estrogenic factors, carbon-fiber-reinforced composites can offer densities and electrical conductivity/resistivity properties close to bone with strengths much higher than metals on a per-weight basis. In vivo bone-marrow tests with Sprague-Dawley rats revealed far-reaching significant osseoconductivity increases from bisphenyl-polymer/carbon-fiber composites when compared to state-ofthe-art titanium-6-4 alloy controls. Midtibial percent bone area measured from the implant surface increased when comparing the titanium alloy to the polymer composite from $10.5 \%$ to $41.6 \%$ at $0.8 \mathrm{~mm}, P<10^{-4}$, and $19.3 \%$ to $77.7 \%$ at $0.1 \mathrm{~mm}, P<10^{-8}$. Carbon-fiber fragments planned to occur in the test designs, instead of producing an inflammation, stimulated bone formation and increased bone integration to the implant. In addition, low-thermal polymer processing allows incorporation of minerals and pharmaceuticals for future major tissue-engineering potential.
\end{abstract}

\section{Introduction}

Foremost advancements are expected in stem-cell/osteoprogenitor/osteoblast tissue-engineering for the next generation of bone implants as a result of new materials available from the stealth-electronic technology aeronautical/aerospace era. Through a better understanding of the microstructure and electron-transfer properties for matter, polymer-based $\mathrm{f}$ iber-reinforced materials can be bioengineered to provide important new materials for broad significant bone implant applications. In the world of materials, fibers are the strongest and possibly stiffest known forms of a substance matter [1]. When combined into an appropriate matrix like a polymer, much of the fiber mechanical-strength properties can be transferred through the bulk material $[1,2]$. Such multiconstituent materials, referred to as composites, have led the way in the aeronautical/aerospace age, primarily as a means to provide stronger lighter structural parts. The basic polymer used for advanced design capability has been a class of thermosetting organic resins that cure by electron freeradical crosslinking $[1,2]$. The thermoset resins generally contain similar interconnecting bisphenyl double-aromatic ring molecules that can be reinforced by chemical coupling with fibers for highly developed mechanical properties $[1,2]$. The bisphenol-derived polymer function was further identified in 1936 through a pharmaceutical study as one of the first synthetic estrogens [3]. Estrogen in turn has a stimulating anabolic effect on bone formation and differentiation of osteoblasts [4-7].

In terms of material biocompatibility, polymers have extensively long hydrocarbon backbone chains and are more similar to carbon-based tissue cells than metals or ceramics. Chemical similarity between neutrally active nonpolar polymer materials and lipid hydrocarbon cellular membrane interfaces are then expected to improve biocompatibility for many new important biomedical applications. Various assorted polymers are currently already used for the transition artificial heart organ, vascular grafts, tendon/ligament repair, guided tissue regeneration, articular joint components, orthopedic medical cements, resorbable scaffolds for tissue growth and resorbable sutures $[8,9]$. In fact, polymer materials are well-known primordial biologic-derived hydrocarbons that were geologically degraded and then recovered by the petroleum industry and separated as pure monomeric 
TABLE 1: Biomaterial properties.

\begin{tabular}{|c|c|c|c|c|c|}
\hline Material & $\begin{array}{l}\text { Density } \\
\left(\mathrm{g} / \mathrm{cm}^{3}\right)\end{array}$ & $\begin{array}{c}\text { Resistivity }^{\mathrm{a}} \\
(\Omega \mathrm{m})\end{array}$ & $\begin{array}{c}\text { Tensile strength } \\
(\mathrm{MPa})\end{array}$ & $\begin{array}{l}\text { Yield strength } \\
(\mathrm{MPa})\end{array}$ & $\begin{array}{c}\text { Modulus } \\
(\mathrm{GPa})\end{array}$ \\
\hline Bone longitudinal-radial hydrated $[8,9]$ & $1.8-2.1$ & $46-150$ & $70-150$ & $30-70$ & $15-30$ \\
\hline Titanium grades $1-4[9,18]$ & $4.5-4.51$ & $10^{-7}$ & $240-550$ & $170-485$ & $104-110$ \\
\hline Titanium-6-4aluminum vanadium alloy $[8,9,18]$ & $4.4-5.0$ & $10^{-8}$ & $860-1103$ & $795-1034$ & $116-120$ \\
\hline Bisphenyl Unidirectional $\mathrm{CF}^{\mathrm{b}}[1,2,18,19]$ & 1.6 & 5 & $780-1850$ & & $145-325$ \\
\hline Bisphenyl Unidirectional $\mathrm{CF}^{\mathrm{b}} 4$-pt. bend $[1,2,19]$ & 1.6 & 5 & $790-1800$ & & $120-255$ \\
\hline $\begin{array}{l}\text { Bisphenyl/QF }{ }^{b} \text { Exp.Uni-woven laminate 4-pt. bend } \\
{[17,19]}\end{array}$ & $1.49(.01)^{\mathrm{c}}$ & 5 & $963(240)^{\mathrm{c}}$ & $774(176)^{\mathrm{c}}$ & $64(14.4)^{\mathrm{c}}$ \\
\hline Bisphenyl 3-D Woven E-Glass 3-pt. Bend X-Y planes [17] & & & $576(129)^{c}$ & $441(75)^{\mathrm{c}}$ & $26(18)^{c}$ \\
\hline
\end{tabular}

${ }^{\mathrm{a}}$ Resistivity $=1 /$ conductivity.

${ }^{b} \mathrm{CF}$ : carbon fiber; QF: quartz fiber.

${ }^{\mathrm{c}}$ Experimental standard deviations in parentheses.

units. Highly advanced biologically derived structural composites developed for military aircraft and aerospace structures are thus now ready for biomedical application.

Free-radical cure thermoset bisphenyl resins are processed at relatively low temperatures, between room temperature and below $200^{\circ} \mathrm{C}$ to produce the hardened cured polymer [2]. So, other organic compounds can be added into the resin before curing and safely processed. Similarities in nonpolar chemical relationships between thermoset resins and many organic therapeutics consequently appear to be a major opportunity toward establishing biocompatible cell/tissue interfaces with implants. For example, due to molecular parallel chemistry interactions nonpolar pharmaceuticals can be blended into resins and cured into a polymer implant interface $[10,11]$ for long-term release and interfacial interactions with adjacent cells and tissue. Nonpolar hydrocarbon additives have subsequently shown the ability to entangle with the main polymer backbone chain to improve matrix toughness properties [11]. A tougher, less brittle composite then aids in making thinner parts [12] which becomes more important for small biomedical devices. In addition, polarized inorganic fillers similar to bone mineral are commonly included in thermoset free-radical cure bisphenyl polymers to improve mechanical/physical properties and control manufacturing process consolidation $[2,13,14]$. Eukaryote mammalian cells then extensively require inorganic calcium and phosphates derived from a large bone source to insulate and seal lipid membrane compartments and establish voltage potentials [15-17]. With a negative membrane potential, cells then have the ability to do work and develop [15-17].

Polymer-matrix fiber-reinforced composites can therefore be engineered for specific tissue performance with potential mechanical properties many times greater than structural aluminum, titanium, or steel on a per-weight basis $[2,18]$ (Table 1 on density, mechanical and electrical conduction properties). Bisphenyl-polymer/carbon-fiber composite electrical-conduction/resistivity properties [9, 17-19] are similar to bone $[9,17]$ with design capability to better simulate conditions favorable to the approximate $-70 \mathrm{mV}$ plasma cell membrane resting potential $[16,17]$ and complex electron-transfer reactions that form the fundamental units for the varied multitude of biologic processes [15-17]. In addition, when compared to metals or ceramics, the modulus or stiffness of polymer composites (Table 1) can be engineered to accommodate stress-transfer conditions with surrounding bone tissue and associated living cells [17]. Modulus mismatch between bone and metals consequently has been a problem with current implant loosening related to stress transfer between the bone and implant [20]. Density in turn is related to modulus through force-interatomicdistance equilibriums [18].

In order to test the hypothesis that bisphenyl-polymer/carbon-fiber composite was more biocompatible than titanium-6aluminum-4vanadium alloy implant material for mesenchymal stem-cell recruitment with osteoprogenitor/ osteoblast proliferation/differentiation, an in vivo rat tibia model [21] was used to measure bone growth by histomorphometry. Statistics were calculated using Microsoft Excel for a $t$ test of unequal variances to compare differences between groups. The marginal level of uncertainty was set at $\alpha=0.05$.

\section{Materials and Methods}

2.1. Materials and In Vivo Animal Test Model. Unidirectional bisphenyl-polymer/carbon-fiber rods $1.5 \mathrm{~mm}$ in diameter were placed for two weeks in vivo using an engineered Sprague-Dawley rat tibia model that has been previously described elsewhere by McCracken and Lemons et al. [21]. As a result, previous tissue slides for $1.5 \mathrm{~mm}$ diameter titanium-6-4 alloy (90\% titanium; 6\% aluminum; 4\% vanadium) material controls were consequently available for comparison [21] to measure percent bone area (PBA) at a specified tibia intramedullary distance from the implant using Bioquant software (Nashville, TN). The titanium alloy screws measured $1.5 \mathrm{~mm}$ diameter $\times 8 \mathrm{~mm}$ length (Walter Lorenz Surgical Inc. Jacksonville, FL). The bisphenyl-epoxypolymer/carbon-fiber composite was composed of $60 \mathrm{vol}-$ ume percent unidirectional carbon fibers with 40 volume percent polymer processed into $1.5 \mathrm{~mm}$ diameter rods using a bisphenol-derived epoxy thermoset resin (Aerospace Composite Products, Livermore, CA). The bisphenyl polymers originate from two interconnecting aromatic rings as 
a bisphenol [2] that has been compared pharmaceutically to the molecular structure estrogen [3].

2.2. Animal Preparation. Animals were maintained according to standards set by the American Association for Accreditation of Laboratory Animal Care following the Guide for Care and Use of Laboratory Animals proposed through the National Research Council (1996). Ten male SpragueDawley rats weighing 350 to $375 \mathrm{~g}$ (4 months old) were obtained for each of the two groups separately comparing PBA surrounding implants for the titanium alloy historical controls and new bisphenyl-polymer/carbon-fiber composite. Two additional rats were also included for an alternate histology imaging characterization for the bisphenylpolymer/carbon-fiber composite. All rats were weighed to the nearest gram. Animals were provided food and water during the experimental procedure ad libitum. The room was maintained at $23^{\circ} \mathrm{C}$, with 12 -hour light-dark cycles.

The animals were anesthetized using Isofluorane from a precision vaporizer inducing at $4-5 \%$ and maintaining at 1 $2 \%$. Rats were also administered intraperitoneal anesthetics with ketamine at $10 \mathrm{mg}$ per $100 \mathrm{~g}$ and xylazine at $1 \mathrm{mg}$ per $100 \mathrm{~g}$. Rats were shaved, scrubbed, and draped to provide a surgical field. A $1.5-\mathrm{cm}$ incision was made on the medialproximal surface of the tibia above the tibial protuberance. Tissue was reflected to expose the flat portion of the tibia below the joint.

Using a slow-speed surgical handpiece with a no. 4 round bur and copious warm saline irrigation, a pilot hole was drilled in the tibia $8 \mathrm{~mm}$ proximal to the tibial cortical bone protuberance. A $1.3 \mathrm{~mm}$ diameter surgical implant twist drill bit was used to create an oblique-transverse osteotomy, traveling through the medullary canal and the opposite cortical shaft. Rather than drilling perpendicular to the bone, an oblique path of implant placement was used to optimize the implant surface area in the canal for each specimen. A no. 6 round bur was used to increase the size of the hole in the medial aspect of the tibia. The osteotomy was irrigated with $20 \mathrm{~mL}$ of warm saline. The titanium-6Al-4V allow screws were placed by hand to engage the opposite cortical shaft but did not engage the medial cortical bone shaft, which had been enlarged with the no. 6 round bur. The bisphenyl-epoxy-polymer/carbon-fiber composite rods were cut to $5.0 \mathrm{~mm}$ lengths, washed, cleaned by ultrasonic vibration and sterilized by autoclave. Autoclave sterilization without ultrasonic cleaning was not used for two alternate composite rat tibia tests which were later sectioned horizontally through the implant at right angles to all other samples for slide analysis. A $1.5 \mathrm{~mm}$ surgical implant twist drill bit was used to create an oblique-transverse osteotomy, traveling through the medullary canal and the opposite cortical shaft as before. The bisphenyl polymer/carbon fiber composite rods were inserted with some pressure and lightly tapped into place. Primary closure was achieved for each animal by approximating the muscle layers with resorbable sutures and closing the skin with surgical staples.

2.3. Histomorphic Analysis. After healing for 14 days, the rats were euthanized with carbon dioxide inhalation and exsanguinated by cardiac incision with sharp scissors. Tibiae were removed, cleaned of soft tissue, and immediately imaged by X-rays and photographs for later characterization and comparison with the histology. Tibial specimens were then fixed in phosphate-buffered paraformaldehyde for a minimum of 12 hours. Subsequent specimens were dehydrated with progressive alcohols under vacuum, cleared with xylene, infiltrated and embedded with methylmethacrylate and polymerized by ultraviolet light. Samples were prepared for light microscopy by cutting and grinding techniques that provided a lateral section of the implant. The final sample thickness was less than 60 um with specimens mounted on clear plastic slides. Slides for the 20-rat group statistical analysis were stained with toluidine blue to identify live bone. One sample from the original polymer composite statistical analysis group was stained with a modified H\&E medium after partially exposing the upper cortical plate to examine the bone inside the tibia canal before solvent dehydration. Sanderson's stain was further used for two additional rat tibia slides not included in the statistical analysis which were prepared with cuts horizontal at right angles through the composite implants.

Percent Bone Area (PBA) was defined as the area occupied by bone within $0.8 \mathrm{~mm}$ and $0.1 \mathrm{~mm}$ of the implant inside the bone-marrow space of the tibia between the cortical bone plates. Previous PBA measurements had been determined with the titanium alloy at $1.5 \mathrm{~mm}$ [21]. A distance of $0.8 \mathrm{~mm}$ was now used as this measure was considered a rough approximation of the physiologic cortical-plate thickness for the Sprague-Dawley rats under study. A distance of $0.1 \mathrm{~mm}$ was also considered as a physiologic estimate for initial osseointegration with basic oxygen diffusion limits. Data was analyzed using BioQuant Software (Nashville, TN). Measurements for the PBA statistical analysis were all completed at 2x magnification with an Olympus microscope on a 19-inch-by-19-inch monitor and were reconfirmed when necessary at $4 \mathrm{x}$ or $10 \mathrm{x}$ visually for accuracy. All stained areas were measured as live bone. Comparisons of different measurements between groups were analyzed using a $t$-test with unequal variances. Results were considered significant at alpha $=0.05$. Imaging was further completed at $20 \mathrm{x}$ and $40 \mathrm{x}$ for more in-depth evaluations.

\section{Results}

3.1. PBA Statistics. After two weeks, major breakthrough significant statistical differences were found when comparing histological tibia intramedullary PBA results for bisphenylpolymer/carbon-fiber composites to titianium-6-4 metallurgy (Figures 1(a) and 1(b)). At a tibia-marrow/implant distance of $0.8 \mathrm{~mm}$, PBA increased from $10.5 \pm 5.3$ to $41.6 \pm 13.9$ when comparing the titanium alloy to the polymer composite, respectively, $P<10^{-4}$. At a tibiamarrow/implant distance of $0.1 \mathrm{~mm}, \mathrm{PBA}$ increased from $19.3 \pm 12.3$ to $77.7 \pm 7.0$ when comparing titanium alloy to the polymer composite, respectively, $P<10^{-8}$. The bisphenyl-polymer/carbon-fiber composite and titianium-64 alloy both increased PBA approximately almost double from 41.6 to 77.7 and 10.5 to 19.5 correspondingly when 


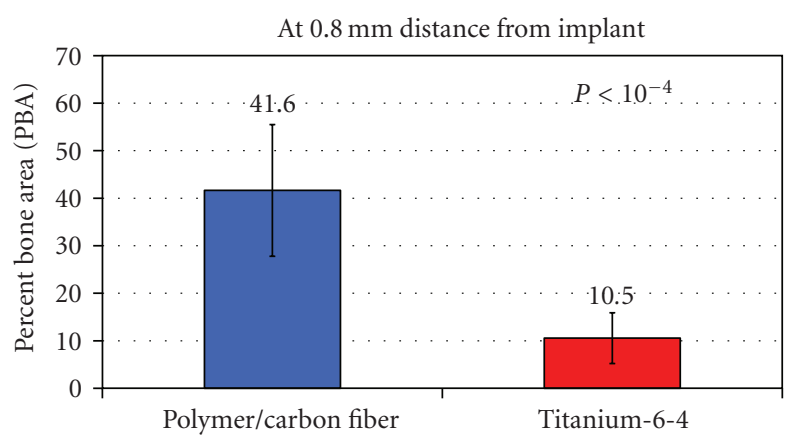

(a)

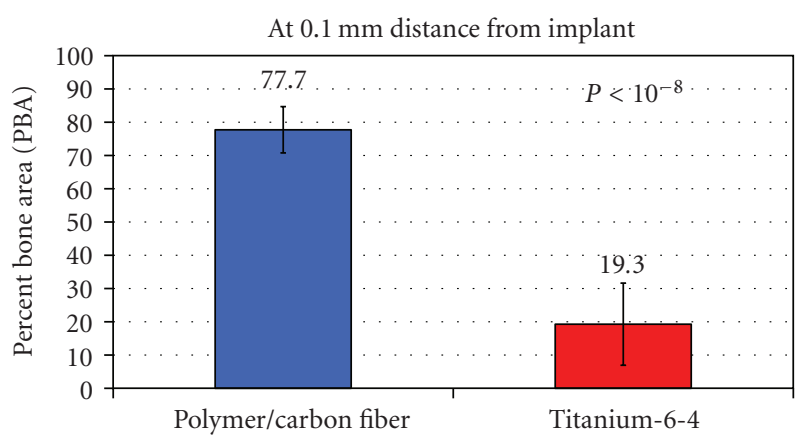

(b)

Figure 1: In vivo implant rat tibia PBA cross-sectional results at $0.8 \mathrm{~mm}$ and $0.1 \mathrm{~mm}$ from the implant surface at midcanal showing highly significant increases for the bisphenyl-polymer/carbon-fiber composite compared to titanium-6-4 alloy. (a) Comparisons for cross-sectional Group Mean PBA out to $0.8 \mathrm{~mm}$ from the implant, $P<10^{-4}$. (b) Comparisons for cross-sectional Group Mean PBA out to $0.1 \mathrm{~mm}$ from the implant, $P<10^{-8}$. The PBA was averaged from both distal and mesial implant surfaces separately at both $0.8 \mathrm{~mm}$ and $0.1 \mathrm{~mm}$. All data presented as the set average \pm one standard deviation error bars. The sample size was 10 for all groups.

comparing results at an implant distance of $0.8 \mathrm{~mm}$ to the much closer distance of $0.1 \mathrm{~mm}$.

3.2. Imaging. As part of the investigation, imaging characterization was completed by gross photography observation in Figures 2(a)-2(f), with X-rays in Figures 3(a) and 3(b), and from histological slides at magnifications ranging from $1.25 \mathrm{x}$ to $40 \mathrm{x}$ in Figures $4(\mathrm{a})-4(\mathrm{~d})$ and $5(\mathrm{a})-5(\mathrm{f})$. The three imaging techniques revealed important biocompatibility potential with significant osseoconductive responses for the bisphenylpolymer/carbon-fiber implants that greatly exceeded the titanium-6-4 alloy standard bone implant screws. Bone growth was stimulated along the entire surface of the bisphenyl-polymer/carbon-fiber implants through the tibia canal bone-marrow space, filled in surgical space between the implant and cortical bone, grew above the normal cortical bone surface levels along the implant and even partially over the end of many exposed composite rods.

Photograph imaging (Figures 2(a)-2(f)) of the $1.5 \mathrm{~mm}$ diameter bisphenyl-polymer/carbon-fiber implants and tissue shows tough collagenous calcifying osteoid in Figures 2(a) and 2(b) that would follow the implant surface above the upper cortical bone plate approximately $1.0 \mathrm{~mm}$ and sometimes also start to grow over the implant end. Fracture of the bone after fixation in Figure 2(c) and before solvent dehydration with embedding for histological section in Figure 4(c) shows that the newly formed intramedullary tibial bone appears somewhat dense and more similar to cortical bone (arrow) rather than trabeculated bone. Separate tests not included in the statistical analysis incorporated minor insignificant bisphenyl-polymer/carbon-fiber fragments as particulate cuttings retained along the implant before surgery which resulted in an overly exuberant collagenous osteoid response extruded on the lower cortical plate over the implant end in Figure 2(d). Dissection of the soft tissue away from the upper cortical plate always became more difficult at the bisphenyl-polymer/carbonfiber implant interface where soft tissue is shown lifted up from the cortical bone and an extremely tough fibrous cuff surrounds the entire implant circumference in Figure 2(e).
An easy dissection around the entire band of tough fibrous cuff tissue that surrounded a bisphenyl-polymer/carbonfiber implant which protruded only minimally above the cortical bone indicates that soft tissue integration is associated with carbon-fiber fragments of fine particulate in Figure 2(f).

$\mathrm{X}$-ray imaging in Figure 3 of the 2 -week rat tibial $1.5 \mathrm{~mm}$ diameter unidirectional bisphenyl-polymer/carbonfiber implant shows an X-ray frontal view in Figure 3(a) with enhanced cortical-like bone growing around the implant. The vertical X-ray bone density of the tibia around the implant could generally approximate and often greatly even surpass the highest levels for cortical edge density. The increased X-ray bone density is particularly apparent from the top as bone grew both through the intramedullary canal and also up the implant outer surface. A typical X-ray lateral view in Figure 2(b) shows cortical-like bone growing along the implant through the tibial canal where bone is not normally physiologically found.

As a comparison reference for low magnification imaging at the $0.8 \mathrm{~mm}$ distance from the implant (Figures $4(\mathrm{a})$ and 4(b)) average mesial and distal PBAs for both the titanium alloy (11.2 mesial and 9.9 distal) and bisphenylpolymer/carbon fiber composite (38.8 mesial and 44.4 distal) were very similar and no significant statistical differences were apparent. The titanium alloy (Figure 4(a)) shows pieces of bone integrating along the implant surface whereas the bisphenyl-polymer/carbon-fiber (Figure 4(b)) shows more extensive coordinated bone formation along the implant surface. High power magnifications from Figure 5 detected some minor fiber fracture and fiber fragments. However, all carbon-fiber exposure to the biologic environment stimulated bone growth immediately at the fiber surface. Some broken fibers were even incorporated directly into the growing bone cell unit. Intense osseoconductivity was accentuated most at surface implant defects for the bisphenylpolymer/carbon-fiber composites (Figure 5(e)). Some light staining measured directly from the slides as bone on a 19-inch-by-19-inch monitor screen that could include higher 


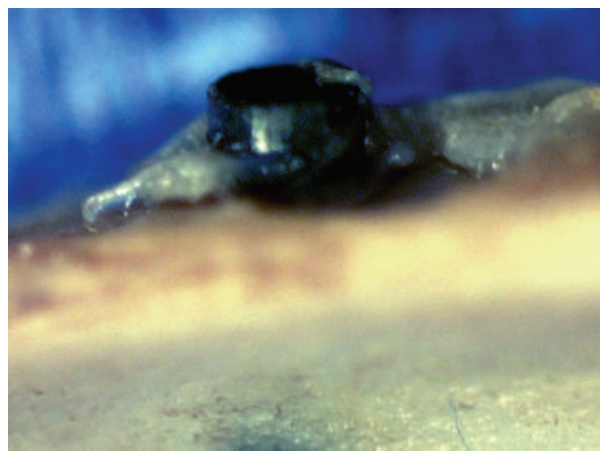

(a)

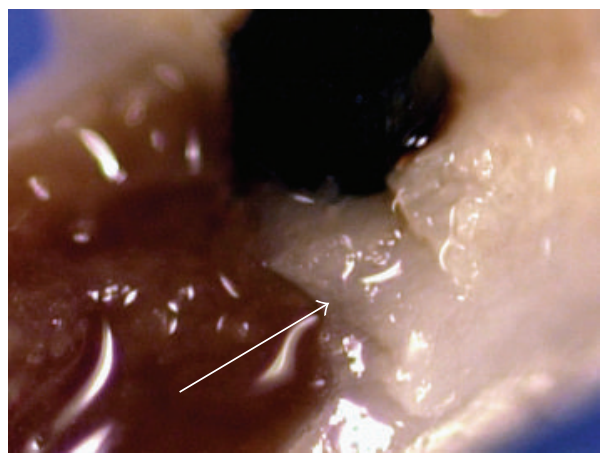

(c)

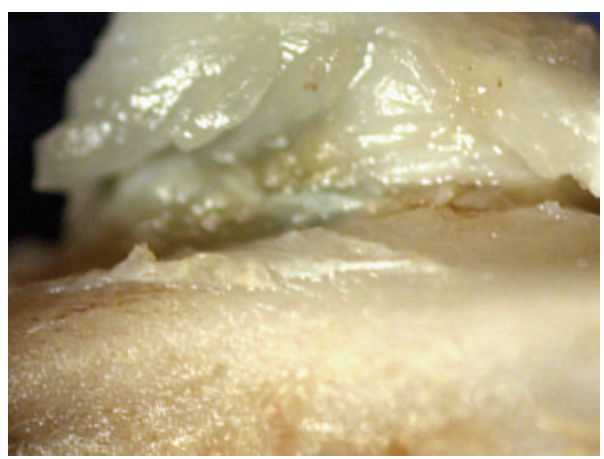

(e)

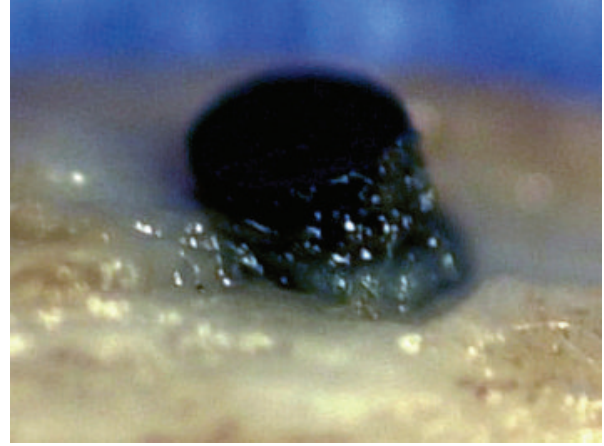

(b)

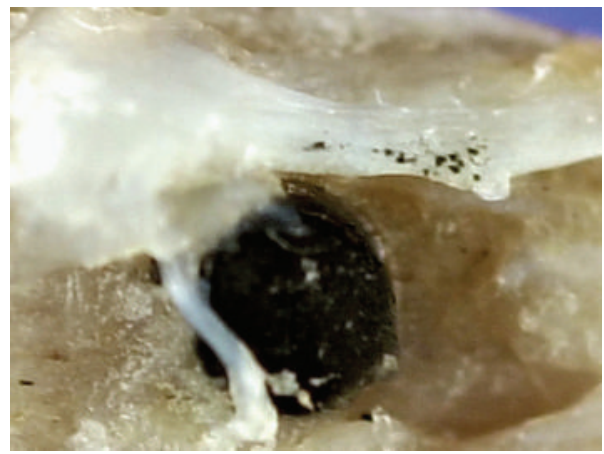

(d)

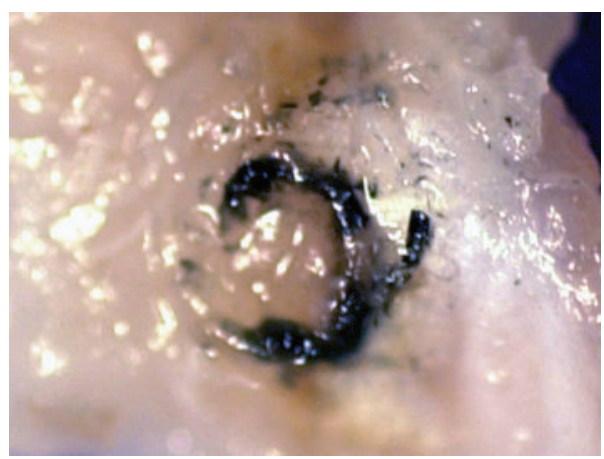

(f)

FIGURE 2: Photograph imaging of $1.5 \mathrm{~mm}$ diameter bisphenyl-polymer/carbon-fiber implants and tissue: (a, b) upper cortical bone plate with osteoid growing up the composite implant surface. (c) Fracture of the bone after fixation. (d) Separate tests not included in the statistical analysis incorporated minor insignificant bisphenyl-polymer/carbon-fiber fragments as particulate cuttings retained along the implant before surgery which resulted in an overly exuberant collagenous osteoid response extruded on the lower cortical plate over the implant end. (e) Dissection exposes an extremely tough fibrous cuff surrounding the entire implant circumference. (f) Tissue dissection of the fibrous cuff provides evidence of carbon fine particulate and fiber fragments.

power confirmation may not be entirely apparent in the reproduced images at lower magnification.

\section{Discussion}

4.1. Material Analysis Accentuated Biocomplex Comparisons. From the rat tibia in vivo intramedullary bone-marrow study comparing titanium alloy to bisphenyl-polymer/carbonfiber composite, material differences were accentuated during extreme osseous formation in the exceedingly low $P$ value PBA study. Osteoblasts/osteocytes are the parenchyma tissue cells involved in the highly biocomplex synthesis and deposition of bone extracellular matrix for ossification that can arise from bone-marrow mesenchymal stem-cell progenitors $[22,23]$. Therefore, possible complex chemistry and biological electron-transfer relationships could be more easily considered with two extreme differences in materials and biological tissue reactions. Through such new emphasized material comparisons, bone appears to be stimulated by bone cell recruitment, proliferation and differentiation from a bisphenyl-polymer/carbon-fiber composite with many potential advantages when compared with the metal 


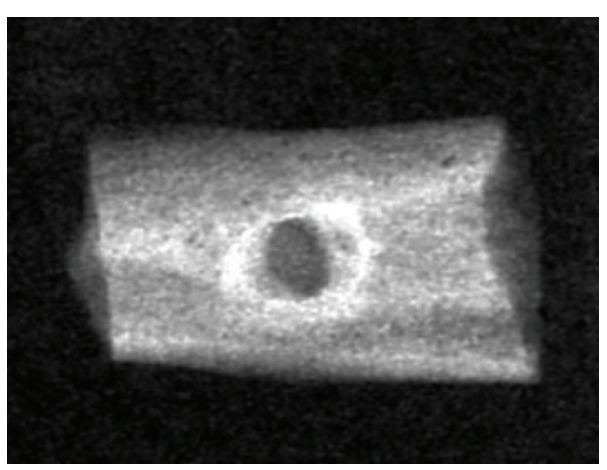

(a)

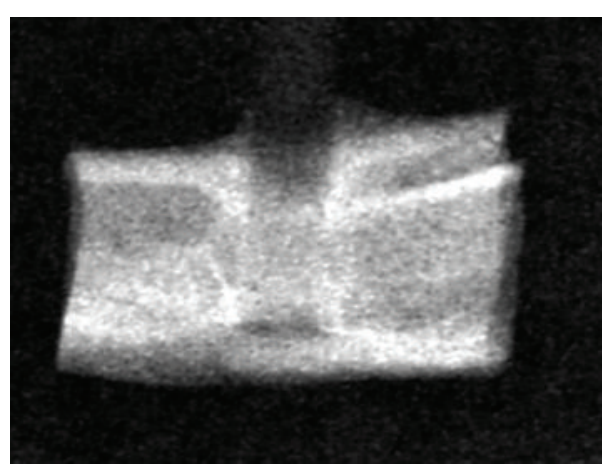

(b)

Figure 3: X-ray Imaging: (a) Frontal view. (b) Lateral view.

TABLE 2: Resistivity ${ }^{\mathrm{a}}$ of different engineering and biological materials $(\Omega \mathrm{m})$.

\begin{tabular}{lcc}
\hline Material & Type & Resistivity \\
\hline Titanium pure & Conductor & $4.2-5.2 \times 10^{-7}$ \\
Titanium-6Al-4V alloy & Conductor & $1.7 \times 10^{-8}[18]$ \\
Bisphenol-polymer/carbon Fiber & Semiconductor & $5[19]$ \\
Composite & Semiconductor & $46[9]$ \\
Bone longitudinal & Semiconductor & $150[9]$ \\
Bone radial & Semiconductor & $0.72[9]$ \\
Physiologic saline & Semiconductor & $3000[26]$ \\
Silicon pure & Semiconductor & $20-80[27]$ \\
Silicon phosphorous Doped & Semiconductor & $100[28]$ \\
Lipid phosphate & & $0.95-1.8 \times 10^{-5}$ \\
headgroup/water interface & Conductor & {$[18]$} \\
Carbon fibers & Insulator & $10^{10}-10^{13}[18]$ \\
Thermoset bisphenyl polymer & & $\sim 10^{-6}-10^{-9}$ \\
General metals & Conductors & {$[18]$} \\
Pure quartz fiber & Insulator & $10^{20}[29]$ \\
\hline a & &
\end{tabular}

titanium alloy. To elucidate on such new important composite material properties, nonpolar and steroid-like polymer factors with possible carbon-fiber biocircuit antioxidanttype electron-withdrawing effects that might occur can be described in further detail.

4.2. Polymer Nonpolar and Estrogen Factors. Nonpolar or hydrophobic polymer surfaces have previously demonstrated superior cell growth and adhesion over more polar or hydrophilic calcium phosphate hydroxyapatite in vitro $[24,25]$. Therefore, another possible biocompatible influence may include nonpolar-nonpolar chemical similarities between the bisphenyl polymer and the bone-marrow bilipid-cell membranes that may promote attractive nonbonded Lennard-Jones parameters and London-type instantaneous-induced-dipole van der Waals dispersion forces. The bisphenyl-polymer backbone will stimulate estrogen steroidlike hormone factors, known to enhance stem-cell differentiation with bone formation [3-7]. Bone-marrow stemcell proliferation further appears possibly linked to the nonpolar aromatic molecules that indirectly produce differentiation effects based on increased cell division, cell packing, and density for more uniform stress transfer as mineralizing cells assume closer relationships to the parenchyma tissue or bisphenol-polymer/carbon-fiber implant. Bisphenyl-polymer nonpolar hydrophobicity for water repulsion may even help favorable biological reactions to proceed at the implant interface by minimizing degrading effects from biological fluids locally. In addition, polymers do not release more polar metallic Lewis acids into biologic fluids for a stable neutral $\mathrm{pH}$ interface.

\subsection{Electrical Conductivity/Resistivity for Semiconducting} Potential. A bisphenyl-polymer/carbon-fiber implant interface with an overall resistivity of $5 \Omega \mathrm{m}$ [19] (Table 2) is about within an order of magnitude for bone mineral longitudinal and radial resistivity of approximately $46 \Omega \mathrm{m}$ and $150 \Omega \mathrm{m}$, respectively [9]. Conversely, titanium alloy resistivity is vastly lower than bone by extensive orders of magnitude at about $10^{-8} \Omega \mathrm{m}$ [18]. Physiologic saline represents another biological comparison to composite resistivity at $0.72 \Omega \mathrm{m}$ [9]. Pure silicon semiconductor has a resistivity that is $3000 \Omega \mathrm{m}$ [26] and phosphorous-doped silicon used in high-energy physics has a resistivity of 20-80 $\Omega \mathrm{m}$ [27]. As another comparison for plasma cell membrane electric biocompatibility performance, in vitro phosphate-head lipid resistivity at the interface between the phosphate head groups and water has been measured at approximately $100 \Omega \mathrm{m}$ [28]. The bisphenyl-polymer/carbon-fiber implant resistivity can further be increased by replacing carbon fibers, 0.95-1.8 $\times$ $10^{-5} \Omega \mathrm{m}$ [18], with pure quartz fibers having a resistivity of $10^{20} \Omega \mathrm{m}$ [29]. Conversely, composite implant resistivity can be lowered by adding conducting metallic particulate with resistivities of about $10^{-6}-10^{-9} \Omega \mathrm{m}$ [18] into the thermoset bisphenyl polymer, $10^{10}-10^{13} \Omega \mathrm{m}$ [18]. Instead of a metal short circuit or polymer/ceramic insulation, the new fiberreinforced composite has semiconducting potential. Related to safe efficient electron-transfer speeds, bone actually is 


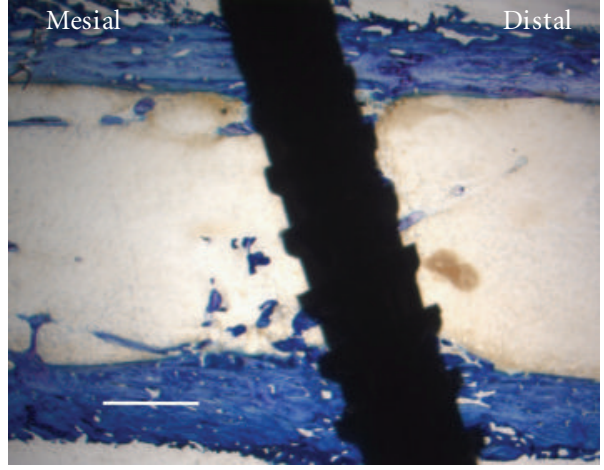

(a)

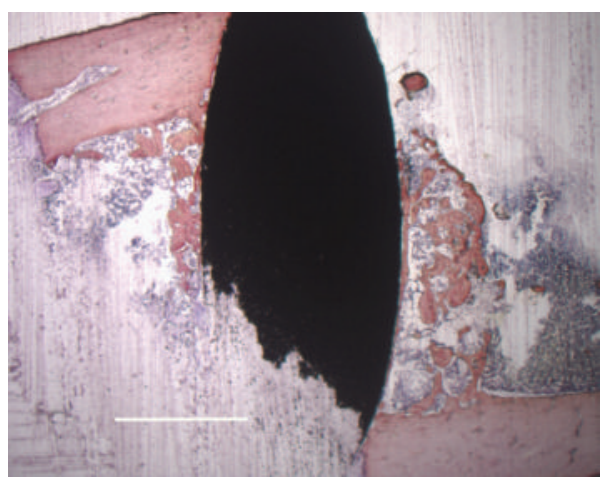

(c)

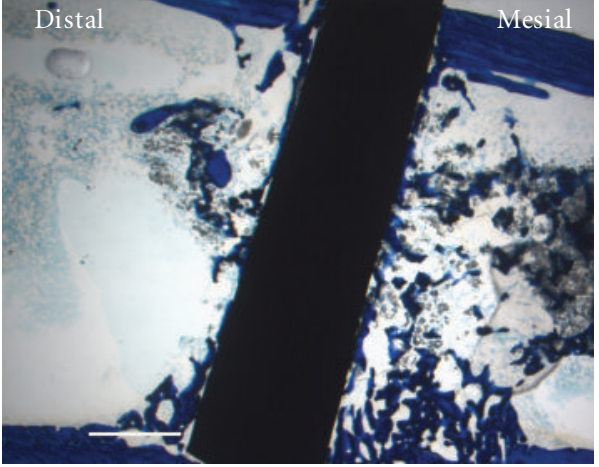

(b)

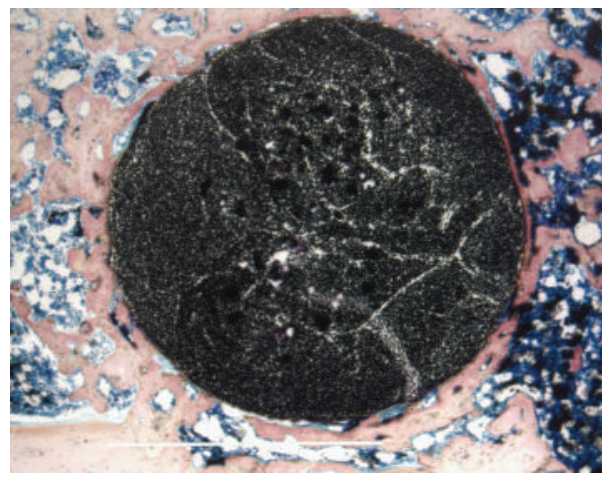

(d)

FIgURE 4: Histological low-magnification imaging with all scale bars approximately $1.0 \mathrm{~mm}$. (a) Lateral section of titanium-6-4 alloy implant as a representative image at the $0.8 \mathrm{~mm}$ implant distance (7.1 PBA from 10.5 PBA average) with toluidine blue stain. (b) Lateral section of bisphenyl-polymer/carbon-fiber composite implant was chosen as a representative image at the $0.8 \mathrm{~mm}$ implant distance (42.3 PBA from 41.6 PBA average) with toluidine blue stain. (c) Lateral intramedullary section of a bisphenyl-polymer/carbon-fiber composite implant from the fractured sample in Figure 2(c) that appears as dense cortical bone before alcohol and xylene solvent dehydration and clearing, respectively, instead provides contrast with a more trabeculated appearance after some osteoid removal using a modified H\&E stain. So, additional osteoid not seen by histology is a probable in vivo structure at some level associated with the bisphenyl-polymer/carbon fiber implants. (d) Horizontal section of a bisphenyl-polymer/carbon-fiber implant from an extra tibial study not included in the statistical analysis provides an alternate view of the cut fiber ends to better appreciate the unidirectional composite with a Sanderson's stain. The original implant diameters in all cases were approximately $1.5 \mathrm{~mm}$.

a well-established piezoelectric tissue that polarizes during bending with a negative electron-transfer potential forming in compression where the healing callus forms in vivo [30], so that a reduced medical fracture is donating electrons and bonds are formed by electron pairing.

\subsection{Carbon-Fiber Conducting Biocircuit and Antioxidant} Effect. Carbon-fiber resistivity at about $10^{-5} \Omega \mathrm{m}$ is electrically conducting that compares at a level similar to metals such as cobalt-base Haynes superalloy [18]. Therefore, when carbon fibers are exposed, electron transfer is achievable at the biologic interface into a relative "sea of electrons" where the polymer insulation creates a micro-biocircuit that may prevent redeposition of potentially damaging free radicals locally back into the original surgical inflammatory zones. Direct carbon-fiber exposure to the bioenvironmental surroundings then might efficiently remove damaging electron energy by a simple electrochemical concentration gradient effect to a more positively charged low electron-level tissue.
Alternatively, by the same electrochemical concentration gradient effect electrons could be effectively donated to reduce fracture or grow tissue such as the fiber cuff stimulated by fiber fragments in Figures 2(e) and 2(f). In addition to surgically induced inflammation from tissue-cell damage, during cellular energy synthesis excess free radicals subsequently further occur during hypoxic metabolic respiration when oxygen is absent [15-17] which is the common state at the implant interface. Without the final oxygen electron acceptor, metabolic acid cannot combine to form the normal physiologic waste product as water [15-17].

4.5. Carbon-Fiber Oxidation for Covalent Bonds. In addition to hydrophobic or nonpolar bisphenyl-polymer cellular biocompatibility, carbon fibers might be highly compatible with bioorganic carbon-based structures. Although bisphenyl-polymer/carbon-fiber composites are considered nonpolar materials, carbon-fiber outer surfaces are polarized by approximately $20 \%$ through oxidation as received thus 


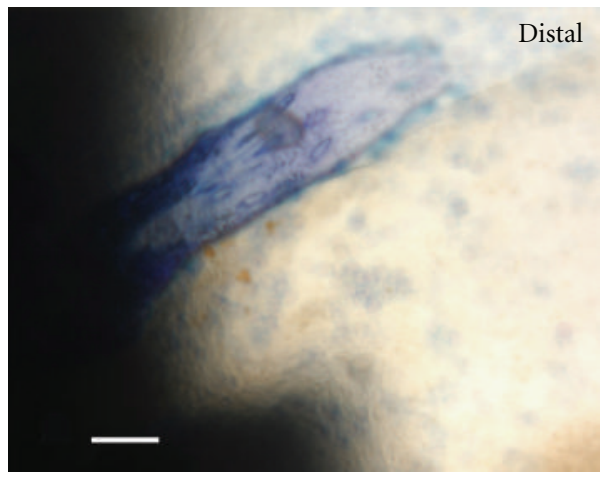

(a)

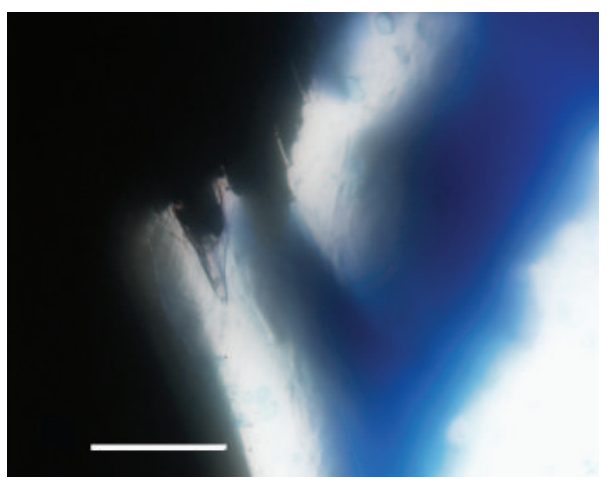

(c)

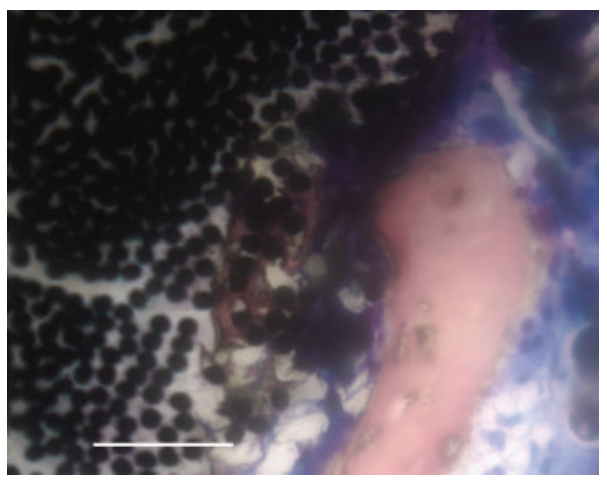

(e)

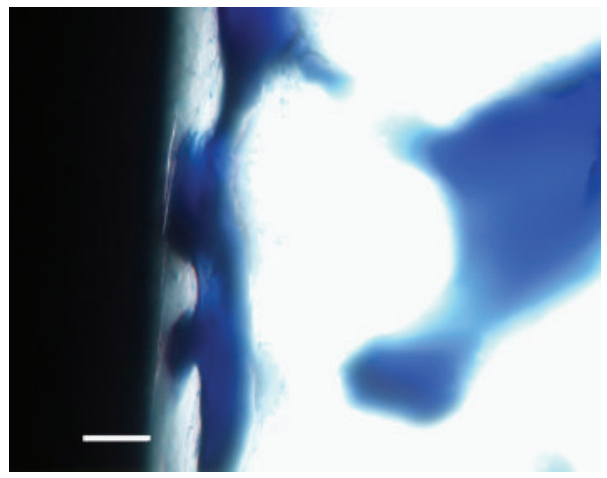

(b)

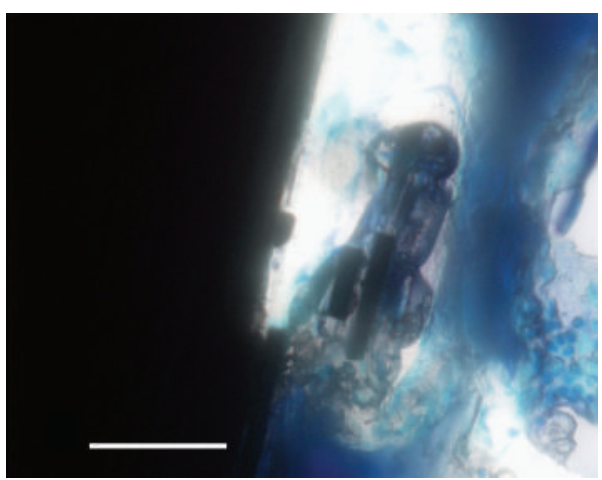

(d)

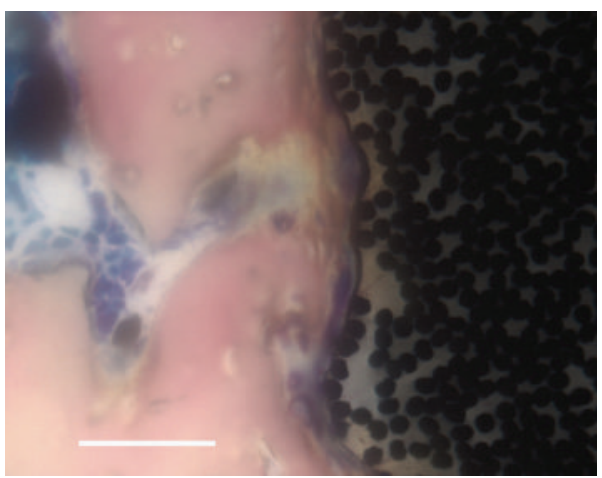

(f)

FIGURE 5: Histological high-magnification imaging with all scale bars approximately $50 \mu \mathrm{m}$. (a) Lateral section of the titanium-6-4 alloy image from Figure 4(a) on distal reveals some implant/bone particle integration using toluidine blue stain. (b) Lateral section of a bisphenylpolymer/carbon-fiber implant exhibits a typical osseointegrated interface with a sophisticated pore-architecture remodeling that may have contained more in vivo osteoid using toluidine blue. (c) Lateral section of a bisphenyl-polymer/carbon-fiber implant presents a fractured composite surface that appears to stimulate bone directly onto an area that represents 2 multiple fiber-fracture ends with toluidine blue. (d) Lateral section of a bisphenyl-polymer/carbon-fiber implant shows an osseointegrating interface with multiple fractured fiber-fragment pieces debonded from the composite and encased in bone with toluidine blue. (e) Horizontal section of a bisphenyl-polymer/carbon-fiber composite implant not included in the statistical analysis expresses calcifications as intense bone integration into the implant surrounding individual carbon fibers of approximate $7 \mu \mathrm{m}$ diameter after removing portions of the polymer matrix utilizing Sanderson's stain. The surface defect is approximately $200 \mu \mathrm{m}$ deeply. (f) Horizontal section of a bisphenyl-polymer/carbon-fiber composite implant also not included in the statistical analysis shows fibers being pulled away from the implant at the bone interface that would necessarily entail polymer degradation or softening, using Sanderson's stain.

forming $\mathrm{R}-\mathrm{COOH}$ or R-COH monolayers [31] to provide the possibility of biologically safe more hydrophilic condensation reactions with living cell membranes through peptide amino acids or lipid fatty acids and sugar or glycerol molecules. Cells may then possibly covalently bond directly to the carbon fibers by either the lipid membrane or glycolipids and glycoprotein linkages with only water as a byproduct. As examples, carbon fibers with polymer that acted as substrates for bone formation at the implant interface (Figure 5(b)) were further incorporated directly 
into the growing bone (Figures 5(c) and 5(d)) anchored new bone around individual fibers (Figure 5(e)) and were even pulled outward away from the polymer at the implant (Figure 5(f)).

4.6. Bone and Material Stress Transfer. Stress-transfer "shielding" related to differences in modulus between the cortical bone (15-30 GPa) and other materials [20] did not apparently play a role in the polymer/carbon-fiber success (145-325 GPa) compared to titanium-6-4 alloy (116$120 \mathrm{GPa}$ ) (Table 1). With both the fiber-reinforced composite and titanium alloy materials displaying moduli of comparative magnitude, stress transfer with bone should be moderately similar. Nonetheless, in vivo static forces were basically in play for the intramedullary bone-marrow implants placed. While not another apparent factor in the present low-stress-transfer study, the density mass/volume relationship $\left(\mathrm{g} / \mathrm{cm}^{3}\right)$ for the bisphenyl-polymer/carbon-fiber composite $\left(1.49-1.6 \mathrm{~g} / \mathrm{cm}^{3}\right)$ is still more similar to cortical bone $\left(1.8-2.1 \mathrm{~g} / \mathrm{cm}^{3}\right)$ than titanium alloy $\left(4.4-5.0 \mathrm{~g} / \mathrm{cm}^{3}\right)$ (Table 1). A lower density material may then improve subsequent biomaterial performance in movement for large implants and proprioception responsive interplay for smaller implants. Also, equivalent density is a probable factor with modulus for uniform stress transfer at the tissue/biomaterial interface, particularly with osseointegration. Further, polymer softening involved in degradation of the composite surface at the bone-tissue interface (Figures 5(c)-5(f)) could improve stress transfer at the molecular and cell levels. Subsequent stress transfer distributed overall more evenly over a greater area through similar modulus/density properties would result in less harmful forces as energy is better dispersed and adsorbed to reduce damage at the molecular and cell levels. Stress transfer then appears to play a possible role in cell differentiation during cell packing at optimum tissue density levels similar to surrounding parenchyma tissue to best reduce molecular damage by equalizing energy adsorption most effectively over a larger volume. Soft polymer/stiff fiber viscoelastic energy damping by the composite $[1,9]$ could subsequently be another factor in idealized stress transfer between the implant and adjacent mineralizing new bone.

4.7. Nitric Acids and Polymer Degradation. Of all acids (97\% sulfuric, $37 \%$ hydrochloric, $97 \%$ phosphoric, and $70 \%$ nitric), nitric acid is the only acid that appreciably reacts with the composite thermoset free-radical cure bisphenyl polymer [17]. In fact, nitric acid reacts many times faster and aggressively with the cured thermoset bisphenyl polymer than the other acids, degrading the polymer entirely into solution while generating an intense green decomposition seen experimentally in our laboratory [17] (Figure 6). Nitricacid polymer greenish decomposition has also been previously described for epoxy aromatic rings produced from bisphenol [32].

By similar nitric-acid chemical interactions with the bisphenyl polymer, acid concentrating enzymes may provide a nitric-acid type of osseointegrating biopathway [17]. Proteins are the most plentiful physiologic buffering systems for acid $[15,16]$ that might subsequently produce safe enzymatic polymer degradation. Moreover along with nitric acid, other strong acids similar to the ones mentioned previously or a weak nitrous acid may further be involved in softening the highly structural crosslinked aerospace thermoset bisphenyl polymer at the surface interface. Related nitricacid ester precursors have already been described biologically as a process that produces a tolerance to nitric oxide and cardiovascular vasodilation [33]. From basic chemistry nitric oxide is unstable with an odd number of electrons [34] and will add to oxygen to form nitrogen dioxide even at room temperature with a decreasing rate with acid by simply dissolving in water [34]. As a consequence, wherever nitric oxide gas forms to reverse the side effects of hypoxic cell mitochondrial free radicals [15-17], nitric acid can ultimately develop [34] whereby negatively charged globular proteins that can further possibly act as enzymes would then be needed for buffering to control pH [15-17]. Nitric acid chemistry with other acids thus appears as a major biochemical need for enzymes that must dissolve difficult biological substrates with aromatic rings. Delocalized free radicals in the protein enzymes should further contribute potential energy with basic components to complement acid degradation. Although toxic breakdown byproducts were considered primarily with the acids and free radicals, the overall abundant bone growth response associated with osseointegration around the carbon fibers suggests that bisphenyl-like molecular structures were further formed as another dominate influence.

4.8. Potential Pharmaceutical and Bone Mineral Polymer Additives. With regard to polymer degradation during periods of oxygen stress at the implant interface, organic therapeutic drugs or inorganic fillers can be designed for incorporation into and release from thermoset polymerbased implants due to low-temperature cures ranging from room temperature to less than $200^{\circ} \mathrm{C}[1,2]$. On the other hand, most metals or ceramics are processed at melting temperatures that can range from approximately 1000 to $3000^{\circ} \mathrm{C}$ [18] so that pharmaceutical or bone mineral incorporation is impossible. Conversely, by lowthermal process, polymer matrix implants can be designed with therapeutic pharmaceuticals to help recruit, proliferate and differentiate bone-marrow mesenchymal stem cells/osteoprogenitors/osteoblasts for optimum successful results. For instance, Triclosan, a broad-spectrum aromatic molecular antimicrobial diphenyl ether with multiple Food and Drug Administration approvals for medical devices, compatibilizes with similar bisphenyl resins to strengthen and toughen the cured polymer while also reducing resin viscosity to improve resin wetting during fiber impregnation $[10,17]$. Related compatible phenyl-aromatic or ring-structured or other organic-type pharmaceuticals may provide numerous biocompatibility avenues for thermoset polymer implant device stem-cell tissue engineering. In fact, aromatic structures compare on a level with amines as a basis for pharmacological therapeutics [35]. Further, ringstructured aromatic molecules are well-known conjugates for estrogens [36] and have been used in the design of 


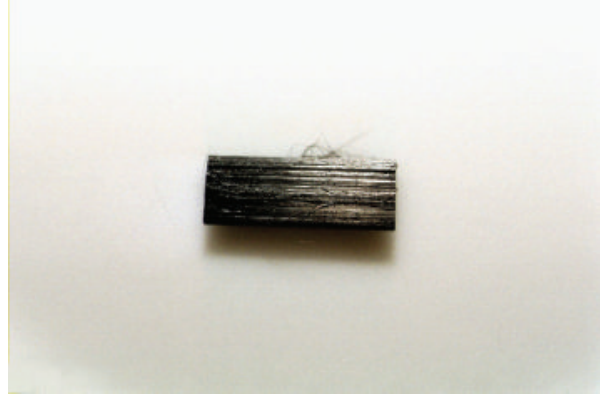

(a)

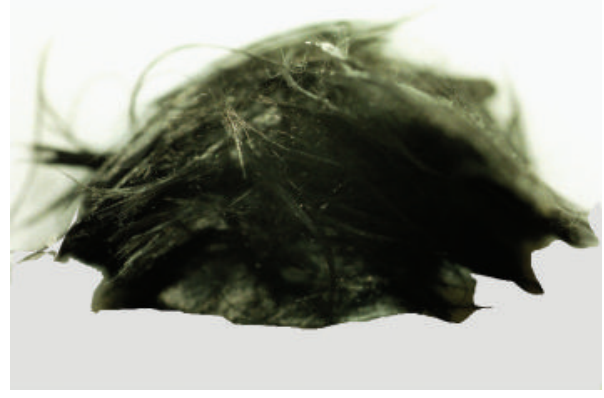

(b)

Figure 6: (a) Typical composite bar exposed to sulfuric acid, hydrochloric acid, or phosphoric acid. (b) Composite bar exposed to nitric acid over a similar time period then washed and dried.

synthetic estrogen-stimulating proteins [37]. Prime examples for thermoset free-radical cure bisphenyl-polymer additive incorporation might include the ring-structured cholesterol derivatives that include vitamin $\mathrm{D}$, anti-inflammatory corticosteroids or steroid tissue-forming androgens and estrogens [15-17]. Further, inorganic bone mineral substitutes can also be cured into the surface ideally engineered for crystallinity or amorphous solubility for long-term or quick release, respectively, for improved osseointegration with respect to continuing stability or immediate bone ingrowth correspondingly.

\section{Conclusions}

In order to prevent fiber loss in reinforced polymer-based composites planned for future use in medical/dental boneimplant devices, fundamental safety factors can now be included during the design with a proper knowledge of material composite micromechanics and macromechanics. Bisphenyl-polymer/carbon-fiber implants provide performance with mechanical properties comparable to current pure titanium grade metals at lower density with one third of the weight. In addition, the bisphenyl-polymer matrix composite further offered a synthetic estrogen nonpolar osseoconductive bone response that provides polymer-type insights into cellular membrane physiology for strong mesenchymal-stem/osteoprogenitor/osteoblast bonemarrow cell recruitment, proliferation and differentiation. Initial concerns for carbon-fiber fragments appear unwarranted now, as all imaging clearly shows increased bone formation associated with loose fibers. Polymeric-insulated carbon fibers appear to stimulate bone through a possible efficient biocircuit electron-transfer antioxidant effect in addition to direct bone integration by possible condensation with biologic molecules. Implant defects, where hypoxic oxygen concentrations would be expected to be low and saturating tissue with mitochondrial free radicals or acids, were associated with deeper implant osseointegration as polymer was even removed and bone surrounded individual electrical conductive carbon fibers. With the ability to specifically engineer polymer-based high-strength carbonfiber-reinforced composites that minimally expose fragments and mimic body tissues, future bone implants should be expected to provide longer patient service with improved lightweight performance.

\section{Acknowledgments}

This paper is funded through the National Institutes of Health Grant no. T32DE14300. Thanks are due to Jack E. Lemons, M.D., Ph.D., Experimental Procedure, Director Laboratory Surgical Implant Research and Implant Retrieval Analysis Center Department of Surgery, School of Medicine and Department of Biomedical Engineering, School of Engineering, University of Alabama at Birmingham; Michael S. Reddy, DMD, DMS, Biological Technical Advice, Chair Department of Periodontology, University of Alabama at Birmingham; Michael S. McCracken, DDS, PhD, Surgery and Surgical Procedure, Associate Dean School of Dentistry and Department of Biomedical Engineering, University of Alabama at Birmingham; Director Patricia F. Lott, Center for Metabolic Bone Disease-Histomorphometry and Molecular Analysis Core Laboratory, University of Alabama at Birmingham, National Institutes of Health Grant no. P30AR46031. Preston R. Beck, epoxy/carbon-fiber composite acid testing, Department of Biomaterials, University of Alabama at Birmingham.

\section{References}

[1] K. K. Chawla, Composite Materials, Springer, New York, NY, USA, 2nd edition, 1998.

[2] S. T. Peters, Handbook of Composites, Chapman and Hall, New York, NY, USA, 2nd edition, 1998.

[3] E. C. Dodds and W. Lawson, "Synthetic estrogenic agents without the phenanthrene nucleus [10]," Nature, vol. 137, no. 3476, p. 996, 1936.

[4] J. B. Lewis, F. A. Rueggeberg, C. A. Lapp, J. W. Ergle, and G. S. Schuster, "Identification and characterization of estrogenlike components in commercial resin-based dental restorative materials," Clinical Oral Investigations, vol. 3, no. 3, pp. 107113, 1999.

[5] L. Gennari, R. Nuti, and J. P. Bilezikian, "Aromatase activity and bone homeostasis in men," Journal of Clinical Endocrinology and Metabolism, vol. 89, no. 12, pp. 5898-5907, 2004. 
[6] R. Okazaki, D. Inoue, M. Shibata et al., "Estrogen promotes early osteoblast differentiation and inhibits adipocyte differentiation in mouse bone marrow stromal cell lines that express estrogen receptor (ER) $\alpha$ or $\beta$," Endocrinology, vol. 143, no. 6, pp. 2349-2356, 2002.

[7] M. N. Weitzmann and R. Pacifici, "Estrogen deficiency and bone loss: an inflammatory tale," Journal of Clinical Investigation, vol. 116, no. 5, pp. 1186-1194, 2006.

[8] B. D. Ratner, A. S. Hoffman, F. J. Schoen, and J. E. Lemons, Biomaterials Science, Elsevier, San Diego, Calif, USA, 2nd edition, 2004.

[9] B. J. Park and R. S. Lakes, Biomaterials, Plenum Press, New York, NY, USA, 2nd edition, 1992.

[10] R. C. Petersen, J. E. Lemons, and M. S. Reddy, "Triclosan antimicrobial for medical/dental devices," in Proceedings of the 2nd Annual South East Tissue Engineering and Biomaterials Workshop, p. 52, Birmingham, Ala, USA, February 2005.

[11] D. Sudhin and D. J. Lobse, Polymeric Compatibilizers, Hanser, New York, NY, USA, 1996.

[12] A. S. Brown, "Revolution in thermoset composites," Aerospace America, vol. 27, no. 7, pp. 18-23, 1989.

[13] R. C. Petersen, J. E. Lemons, and M. S. McCracken, "Micromechanics for fiber volume percent with a photocure vinyl ester composite," Polymer Composites, vol. 28, no. 3, pp. 294-310, 2007.

[14] J. V. Milewski, "A study of the packing of milled fibreglass and glass beads," Composites, vol. 4, no. 6, pp. 258-265, 1973.

[15] L. Sherwood, Human Physiology, Thompson, Belmont, Calif, USA, 5th edition, 2004.

[16] B. Alberts, D. Bray, J. Lewis, M. Raff, K. Roberts, and J. D. Watson, Molecular Biology of the Cell, Garland Publishing, New York, NY, USA, 3rd edition, 1994.

[17] R. Petersen, Micromechanics \& Fiber-Reinforced Composites in Biomedical Research, VDH, Saarbrücken, Germany, 2008.

[18] W. D. Callister, Materials Science and Engineering, John Wiley \& Sons, New York, NY, USA, 1997.

[19] Y. Ramadin, S. A. Jawad, S. M. Musameh et al., "Electrical and electromagnetic shielding behavior of laminated epoxycarbon fiber composite," Polymer International, vol. 34, no. 2, pp. 145-150, 1994.

[20] G. Ryan, A. Pandit, and D. P. Apatsidis, "Fabrication methods of porous metals for use in orthopaedic applications," Biomaterials, vol. 27, no. 13, pp. 2651-2670, 2006.

[21] M. McCracken, J. E. Lemons, F. Rahemtulla, C. W. Prince, and D. Feldman, "Bone response to titanium alloy implants placed in diabetic rats," International Journal of Oral and Maxillofacial Implants, vol. 15, no. 3, pp. 345-354, 2000.

[22] A. J. Friedenstein, R. K. Chailakhyan, and U. V. Gerasimov, "Bone marrow osteogenic stem cells: in vitro cultivation and transplantation in diffusion chambers," Cell and Tissue Kinetics, vol. 20, no. 3, pp. 263-272, 1987.

[23] J. J. Minguell, A. Erices, and P. Conget, "Mesenchymal stem cells," Experimental Biology and Medicine, vol. 226, no. 6, pp. 507-520, 2001.

[24] S. A. Redey, M. Nardin, D. Bernache-Assolant et al., "Behavior of human osteoblastic cells on stoichiometric hydroxyapatite and type A carbonate apatite: role of surface energy," Journal of Biomedical Materials Research, vol. 50, no. 3, pp. 353-364, 2000.

[25] P. Zanchetta and J. Guezennec, "Surface thermodynamics of osteoblasts: relation between hydrophobicity and bone active biomaterials," Colloids and Surfaces B, vol. 22, no. 4, pp. 301307, 2001.

[26] D. Halliday, R. Resnick, and J. Walker, Fundamentals of Physics, John Wiley \& Sons, New York, NY, USA, 4th edition, 1993.
[27] B. S. Avset, "Evaluation of silicon diodes made on a variety of high-resistivity phosphorus-doped substrates," Nuclear Instruments and Methods in Physics Research A, vol. 385, no. 1, pp. 137-144, 1997.

[28] G. L. Jendrasiak and R. L. Smith, "The interaction of water with the phospholipid head group and its relationship to the lipid electrical conductivity," Chemistry and Physics of Lipids, vol. 131, no. 2, pp. 183-195, 2004.

[29] 29. Kossuth, Quartzel Fused Quartz Textiles, Saint Gobain, Advanced Ceramic Division, Quartz Technology Department, 77793 Nemours Cedex, France. Paris, April 2/1/002E, 1998.

[30] E. Fukada, "History and recent progress in piezoelectric polymers," IEEE Transactions on Ultrasonics, Ferroelectrics, and Frequency Control, vol. 47, no. 6, pp. 1277-1290, 2000.

[31] D. Youxian, W. Dianxun, and S. Mujin, "A study of the surface of carbon fiber by means of X-ray photoelectron spectroscopyIII," Composites Science and Technology, vol. 30, no. 2, pp. 119126, 1987.

[32] J. A. Bornmann and C. J. Wolf, "Reaction of nitric acid with a solid epoxy resin," Journal of Polymer Science, vol. 22, no. 3, pp. 851-856, 1984.

[33] H. Burkhard and H. Schroder, "Nitrate tolerance is specific for nitric acid esters and its recovery requires an intact protein synthesis," Biochemical and Biophysical Research Communications, vol. 1998, no. 1, pp. 232-235, 1998.

[34] S. S. Zumdahl, Chemistry, D. C. Heath and Company, Lexington, Mass, USA, 1993.

[35] A. G. Gilman, L. S. Goodman, T. W. Rall, and F. Murad, The Pharmacological Basis of Therapeutics, Macmillan Publishing Company, New York, NY, USA, 7th edition, 1985.

[36] S. Christoph and F.-J. Meyer-Almes, "Novel fluorescence based receptor binding assay method for receptors lacking ligand conjugates with preserved affinity: study on estrogen receptor $\alpha$," Biopolymers, vol. 72, no. 4, pp. 256-263, 2003.

[37] R. Kasher, B. Gayer, T. Kulik et al., "Design, synthesis, and evaluation of peptides with estrogen-like activity," Biopolymers, vol. 76, no. 5, pp. 404-420, 2004. 

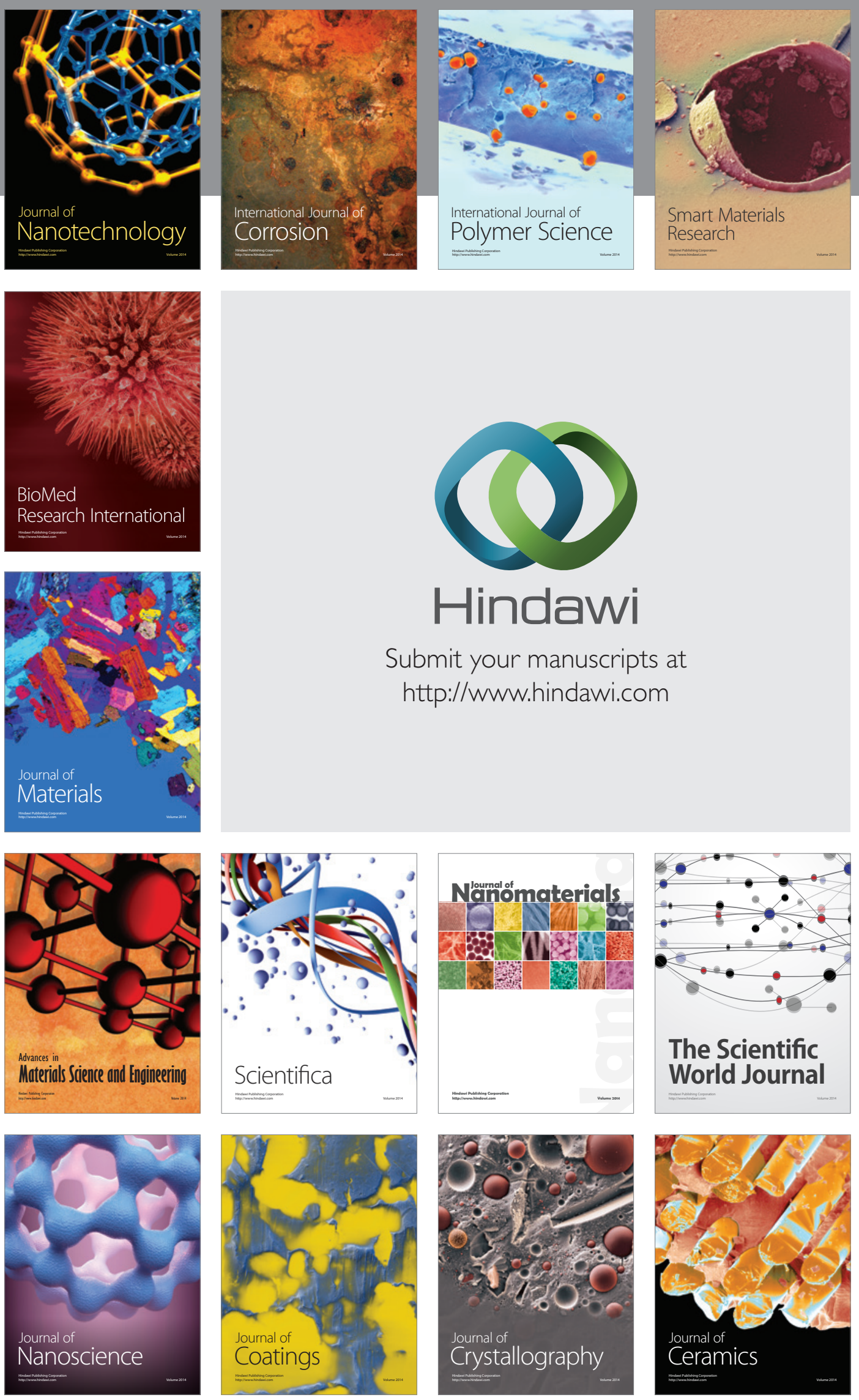

The Scientific World Journal

Submit your manuscripts at

http://www.hindawi.com

\section{World Journal}

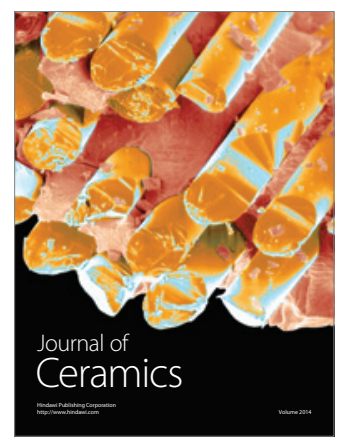

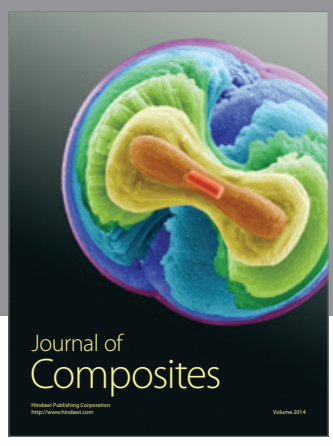
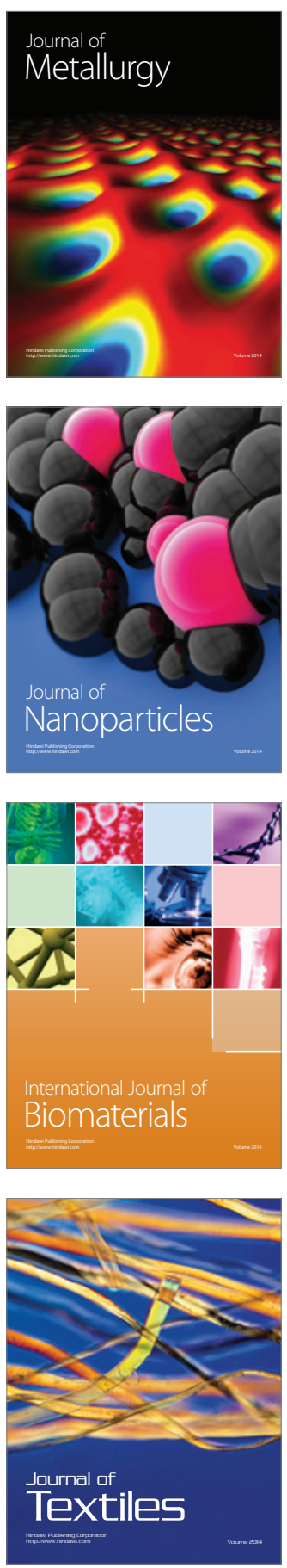